\title{
GI-MS46-04 | Macromolecular Crystallography at MAX IV
}

Gonzalez, Ana (MAX IV, Lund, SWE)

BioMAX is one of the first operational beamlines at the MAX IV facility. It is a multipurpose beamline for macromolecular crystallography, capable of conventional data collection, high throughput and microcrystal applications. The beamline equipment includes a double crystal LN2 cooled monochromator, KB mirrors, a Beam Conditioning Unit, a MD3 diffractometer and a 16M Dectris Eiger detector. Samples are automatically mounted with a ISARA sample exchanger. The beamline uses MXCUBE3 [1] as the data collection interface. Data are processed automatically and the results are displayed to the users with ISPyB [2].

BioMAX has been open to general users since 2017.It has been used by hundreds of users from different countries and it also supports in-house projects to develop serial crystallography and a fragment-based drug discovery facility (FRAGMAX). A second beamline, MicroMAX, with a beam size between 1 and 10 microns, will be dedicated to micron-size beam and crystal sizes, and include serial crystallography and time-resolved applications. MicroMAX has secured funding and first user experiments are planned for 2022.

[1] Delagenière, S. et al (2011) Bioinformatics, 27, 3186-3192.

[2] Mueller, U. et al (2017) Synchrotron Radiation News, 30, 22-27. 